\title{
Demographics Supplemental Qualifiers Dataset
}

National Cancer Institute

\section{Source}

National Cancer Institute. Demographics Supplemental Qualifiers Dataset. NCI

Thesaurus. Code 6147200 .

A dataset containing supplemental information, specifically non-standard variables, to parent records in the demographics domain. 